\title{
Against Universalism in Biosemiotic Theories
}

Claudio J. Rodríguez H.

Palacký University Olomouc, Department of General Linguistics

Received: May 2020; Accepted: June 2020

Abstract: The frontiers of biosemiotics are inconspicuously blurry. This is a feature and not a bug of the discipline in that it allows us to ask questions beyond certain boundaries, enriching both our knowledge beyond semiotic theories and the possibility of covering new ground through them. Yet, explanatory power should be something of a concern for biosemioticians looking to plant flags around different heights. The paths cleared by backwoodsmen should hold up to scrutiny, and in order for biosemioticians to examine these paths, some of the features of semiotic theory should work as reminders of what the aim of semiotic theory is.

This paper will explore one particular issue when it comes to building biosemiotic theories, namely, the idea that the semiotic comprises a universal and basal quality in a hierarchy of elements assumed to give rise to other, more complex things. The metatheoretical problem at its core will be defined as the unnecessary expansion of semiotic attributes in order to give them enough explanatory power to either provide semiotic theories of everything or give a semiotic basis to theories that do not, in principle, require it.

Keywords: metatheory, semiotic theories, scientific explanations

\section{INTRODUCTION}

There are issues of metaphysics that go overlooked, and there are ontological commitments that are taken for granted. There are theoretical commitments we make without having a clear notion of what it is we have signed up for, and there are theoretical stances that are oblivious to themselves. Semiotics-and particularly biosemiotics as one of its theoretical extension-largely deals with all those issues, as many other disciplines do, and the interdisciplinary nature of such a research program doesn't give us much respite. Now sure, the doom and gloom of such a presentation of issues is not really so terrible after all: There is a vibrant, productive community of biosemioticians working daily on theories of biological meaning and their tenets.

This paper is both about the metaphysics involved in theories of biosemiotics and the metatheoretical aspects of being involved in such

situations. We will cover the first part with a critique to the philosophical claims about the universal scalability of semiotic concepts, leading to the second part, where we will consider how issues with ontological claims are crucial in the construction of some semiotic theories.

Now, this is mostly a negative account of what semiotic theories are capable of doing in the line of deflationary metaphysics, but the concern goes beyond that dispute. As far as theories go, we are bound to talk about what exactly counts as a semiotic theory and what lies beyond the tangible aspects of the theory. There have been palpable consequences of a less-than-clear approach to the boundaries of the theory, the most important of which is the institutional detachment of code biology with regards to biosemiotics (as a Peircean enterprise). While I do not intend to solve such issues, I think it is important to approach the topic, even if broadly. If biosemiotics is to be taken as a base-level theory for semiotics, can its theory be extended to prebiological conditions? Would biosemiotics entail an extended form of explanatory power over phenomena that seemingly does not pertain the semiotic? We will explore how positive arguments are crafted for the possibility of a more 
universalistic account of biosemiotics and to what degree these arguments make sense. We will further question how biosemiotics as a discipline regulates philosophical perspectives that attain to such expansions.

\section{POSITIVE ARGUMENTS FOR UNIVERSALISM}

Semiotics is, generally speaking, a hard beast to tame. All our general concepts lead to discussing what is otherwise beyond the realm of possibility for non-semiotic approaches in that a serious discussion about meaning-making tends to be conceptually marginalized from biology (and beyond). However, semiotics perseveres despite this displacement, and its theories extend or oppose views on evolution, chemical interaction, neurology and so on in order to make the point that meaning-making processes, whatever those may be, are an inextricable part of the fabric of the universe.

One of the first assumptions we have to make in this paper is that biosemiotic theories try to follow a more fundamental understanding of semiosic processes, and that the extension of the biosemiotic towards the non-semiotic usually requires a biosemiotic basis to work. ${ }^{1}$ A precis required here is remembering that biosemiotics itself is an extension of semiotic theory, and that it is on this particular extension of theory as a grounding platform that I wish to speak about the potential of universalism in semiotic theories. What I mean by universalism in this context is the idea that semiotic theory, be it through semiotic properties or semiotic causes, is capable of describing what traditionally lies beyond the threshold of what counts as semiotic $^{2}$ at a basal level. That is, physical, emergent and interactive systems are assumed to depend on some semiosic capabilities or semiotic properties to work at all.

Universalism is represented by a number of trends in biosemiotics, including the controversial physiosemiosis, the evolutionary aspect of semiosis and emergent accounts of meaning based on basal semiotic properties. Positive accounts here refer to semiotic theories that argue in favor of semiotic properties or causes at basal levels as universal constants. These theories do not necessarily endorse pansemiotism, and this is important to keep in mind. Physiosemiosis as a theoretical object is said to be compatible with an anti-pansemiotic view, at least in the variety espoused by Deely (2001), but pansemiotic theories can also be grouped within what we are calling universalist theories or the extended model of semiosis. We will explore this concept further.

\section{THE EXTENDED MODEL OF SEMIOSIS}

If semiosis is generally to be taken as the action of signs, the idea of an extended model lies in the corollary that signs are effective where interpretation is not a clear biological imperative. Biosemiotics hinges on the identification of semiosis with living systems, because it is assumed that acts of interpretation, or sign manipulation for that matter, are only possible when agents are involved within a spectrum of relevance and usefulness for self-sustainability. At the simplest levels of biological activity, there is a hearty debate on how meaning-making takes place (if at all), where semiotic guidelines become fuzzy, but more or less identifiable within the assumed coextension of life and semiosis. The less controversial take here is that the cell is the most basic semiotic system (Barbieri 2007; Kull et al. 2011), this being the orthodox view of the extension of biosemiotics, notwithstanding philosophical differences about what makes these systems semiotic and how to treat them. What I am referring to as the extended model lies on the following suppositions: That life is differentiable from non-life; that non-life predates life; and that semiosis is active in non-life.

A general problem with this conceptualization is that a fine-grained distinction between life and non-life is generally not feasible, so the orthodox view certainly allows for the exploration of these boundaries, as in Sharov and Vehkavaara (2015). The differentiation and semiotic ligature between both "worlds" is coarse, so the extended model we are referring to needs to go well past the boundaries of the more orthodox threshold to be identifiable as such. That is, emergent accounts of semiosis do not necessarily need to be grouped within the extended model, but there is a gray area that has not been fully cleared up (conceptually, that is) by biosemiotic research.

The extended model entails that semiosis can be found, even if partially, within non-life, but unlike proposals on protosemiosis, the idea is not just considering semiosis as an emergent at a level of composition close to that of the level of non-life directly below the semiotic, but as a more generalized phenomenon. To the previous assumptions we have to add something we mentioned earlier, namely the inclusion of either semiotic properties or causality as basal universals (though their actual theoretical appearance will vary from position to position). The point is, it is not enough to claim a semiotic stake within non-life, because we can have interpreters interacting with non-life in a fully semiotic fashion. In order to have an extended model we have to assume that these semiotic properties are a natural disposition, so to speak. My inclination is to describe these as semiotic universals, and making a distinction, once again, with the theories of physiosemiosis, which can clearly be included here, but do not comprise the totality of theories that make use of the extended model. While the extended model may

\footnotetext{
1 An argument can be made following the opposite direction, that is, that seeing the emergence of the semiotic from the non-semiotic expands semiotic theory towards the semiotic, but for the purposes of this paper, we will consider both directions within a general idea of an "extended model of semiosis."

2 On the concept, Rodríguez Higuera and Kull (2017) have a useful summary of positions and definitions.
} 
not necessarily inflate to give semiotic explanations to every phenomenon (à la pansemiotics), it conceptually extends semiotic explanations to the non-semiotic world wholesale, both on the edges of the presemiotic and the postsemiotic, to give these areas a name.

\section{SEMIOTIC PROPERTIES AND CAUSES}

The subset of physiosemiotic theories can be understood, in a general manner, as the argument that physical systems are capable of entailing semiosic relations. Semiotic properties and causes may be invoked, but the exact conceptual approach is constrained to the type of systems that count as semiotic. We do not need to approach semiotic properties as physical properties, if we feel the need to invoke them, ${ }^{3}$ opening up space for an odd type of semiotic dualism. Moreover, we can operate at the level of neutral monism. All in all, if we are ready to expand semiosis below the assumed threshold and the gray boundaries within, we must have two things: a need for semiosis at those levels; and a way to present it there.

Beyond the reasons why one would find such a model desirable, its presentation has more to do with the fact that physical systems are not necessarily the limit of the extended model. That is, having semiotic causes operating at the same level as physical causes does not entail that semiotic causes are physical. In other words, an extended model of semiosis only needs to have some form of semiosis earlier than the advent of life (or whatever its boundary conditions are) in whatever form without being limited to purely physical systems as coined in a non-biotic theoretical form.

As far as an extended model of semiosis is concerned then, if semiosis is to be coined as a property or cause that, either in conjunction with others or single-handedly, causes novel things to arise-things we currently identify as semiotic-then what we need for it to work is a conceptual background to claim that the basal semiotic predates the known semiotic in some capacity, i.e., some heretofore unidentified semiotic cause makes biosemiosis actual.

\section{SEMIOTIC CAUSES GIVING RISE}

\section{TO PREFIXED SEMIOSES}

The positive accounts of why we would need some semiotic cause to give rise to semiotic phenomena that we usually account for (as semiotic) can be further characterized as universalistic in the sense that their explanatory power is extremely ample at both base and high complexity levels. In that sense, the idea of the extended model implies that semiosis should be basal to the description of the elements that populate our world. While teleology does not need to be accounted for, it seems to be the case that semiotic causes are directed towards more (and perhaps more complex) semiosis. The possibilities depend on what exactly the theory is trying to achieve, but semiotic causes should be a prerequisite for an extended model in the vein we have been mentioning, to the degree that we can say that semiosis is one of the key elements in the composition of the universe below the traditional threshold.

Prefixed semioses, such as biosemiosis, zoosemiosis, cultural semiosis and so on, can be seen within an interconnected network of sign usage. These, it may be contended from all areas of semiotics, have as their organic source the basic semiosic capabilities of organisms and the growth of signs as two principles, one advocated by biosemiotics and the other, by general Peircean semiotics. The extended model of semiosis seems to have little bearing on this, but it should work as a guiding principle, if not on teleological argumentation, at least on the desired extension of semiosis from basic formulations towards complex cultural (and perhaps post-cultural) semiotic formations.

It would seem that the extended model can bring further support to the idea that semiosis is an evolutionary phenomenon by creating a robust background that affirms its teleological constitution. At the same time, it is because of the apparent connection between semioses, either as processes that follow the same patterns or as more specific forms of semiosies scaffolded on earlier ones, that the extended model can be seen as actually "extending" anything at all. Since there is an assumed organic connection between the different levels (or forms) of semiosis, the extended model can extend below the assumed threshold in an attempt to explore how these connections are brought about in the first place.

Now, even though the distinction between talking about semiotic properties and semiotic causes can have a lasting impact on how we model semiosis within the extended model, we will limit ourselves to treating both possibilities as part of a general context of metaphysical extension-the extended model of semiosis is a theoretical articulation of how positions become universalistic by virtue of their coverage within semiotics, but few options have been philosophically fleshed out to identify them further.

\section{THE EXTENDED MODEL, IN A NUTSHELL}

In our wish to simplify things, what we have been calling the extended model of semiosis can be described in opposition to the standard view on where the semiotic threshold is located. Taking biosemiosis as our standard stance, that is, following Sebeok's idea that life and semiosis are coextensive, we assume that the standard model of semiosis depends on this condition while being neutral to the possibility that presemiotic or protosemiotic phenomena may take place in a grey area between the non-biotic and the biotic world. The extended model of semiosis would simply extend semiosis proper to what is currently considered as non-semiotic, remaining

3 Peircean realists would likely not approve of such an approach. 
also neutral about the type of semiosis that takes place within it. Such an extended model could be capable of arguing for the evolution of semiosis into what is more generally accepted as such, as well as expand ontologies into accepting some form of radical semiosis as a necessary constituent of the universe.

In what follows we will examine how some theories can actually be described within the general schema of the extended model of semiosis as we have conceptualized it so far.

\section{THE UNIVERSALISTIC VISION OF SEMIOTIC THEORIES}

Semiotic theories come in different shapes, complexities and languages, and distinguishing their power and interaction with other theories may not always be a matter of direct comparison. Take, for instance, Koch (1986) and his idea of an evolutionary cultural semiotics with a strong prebiotic component to it. This is a sort of theory that, by virtue of its wide range of comprised phenomena, we can call universalistic and neatly fit within the idea of an extended model of semiosis. Generally speaking, Koch's view on semiotics is teleological, and starting from the bottom semiosis leads the development of other levels, all in one chain where cultural semiosis becomes the endpoint and its most complex layer.

Theories on physiosemiosis, as we have mentioned earlier, have a more or less similar extension without necessarily assuming the evolutionary component of semiosis into complexity. Deely's Peircean semiotics is an example of a comprehensive system that gives semiosis strong explanatory powers for higher levels of complexity with an explicit commitment to a philosophical paradigm. In that way, notwithstanding physiosemiotic claims, we find that Deely does conform to an extended model of semiosis by supporting metaphysical claims on the extension of semiosis as prebiotic and with a distinct temporal dimension such as vis a prospecto (Deely 2012), the reverse temporal causality that appears to come about because of the action of signs.

Theories supporting pansemiosis can also, as mentioned earlier, be grouped within the idea of an extended model of semiosis, but the semiotic premises are much stronger in their outcome. Pansemiotism is a bona fide case of a full-blown universalistic theory of semiotics that has the power to explain every phenomenon as at least partially semiotic. In the case of an actual (yet conservative) pansemiotic view, Salthe $(2012,54)$ makes the case for a reduced form of semiosis within the abiotic realm that plays within a hierarchized view of the world, going from the physical to consciousness. The issue of the gray area surrounding the lower threshold of semiotics is not simple to avoid, but the extension of this sense of semiosis, or protosemiosis as Salthe calls it, plays a much more expanded role than, say, the protosemiosis of Sharov and
Vehkavaara (2015), and so while I see the latter as operating within the more traditional framework of biosemiotics, the former seems to represent a more specific case of the proposed extended model of semiosis.

Information seems to play a crucial role in presenting the possibility of the extended model because, following Salthe's work, it can be conceptualized as state constraints in physical systems that can in turn derive in meaningful states (Salthe 2008, 134-135). Since semiosis would be meaningfulness in action, part of the semiotic relation would lie outside of the spectrum of cognition. This is insufficient for the extended model in that a sign relation partially entailed by an abiotic object does not imply anything particular about the sign relation itself. Salthe $(2008,135)$ uses a ridge in the pathway of running water as an abiotic example of an informational constraint. However, Salthe $(2007,208)$ intends to generalize semiotic mechanisms below the standard threshold via the hierarchies he conceptualizes for the world not only by taking the (common) view that a semiotic relation can be partially instantiated biosemiotically, but by assuming that abiotic interactions (such as those in chemistry) can be characterized independently by adding a concept of interpretation that operates independently from any sort of cognition. The thinking would go, we may assume, in the following way: ${ }^{4}$ physical constraintssuch as a chemical reaction-produce information, and this information is meaningful by itself because there is a reaction taking place that results in something. As long as the reaction is somehow causally connected, its information is fair game as far as his idea of semiosis is concerned. If we allow some forms of causality to produce information independently from biotic processes, and having said information steer towards more complex forms of information (interpretation, semiosis), then the theory becomes a fair representation of what we have been coining as the extended model of semiosis.

Now, the Peircean bent seems to be a common ground between these theories, but that does not imply that all usages of Peirce end up transformed into a fitting description of the extended model. Instead, frameworks such as cybersemiotics seem to be more limited in their explanatory power while still being stronger than the more standard biosemiotic view.

With that brief overview of which actual theories of semiotics would fit in our idea of an extended model, we need to ask ourselves a couple of questions. Namely, can theories of semiotics have that level of explanatory power? And why would we need them to act that way in the first place?

\section{CAN SEMIOTIC THEORIES BE THEORIES OF EVERYTHING?}

Let's try to answer the second question first in the same vein we explored it in the beginning. Why would we ever

4 Taking mostly from Salthe $(2007,2008)$. 


\section{Rodríguez}

need semiotic theories to have an explanatory power as that of the extended model? The answer may go in many different ways, but committing to some semiotic entities cannot be simply dismissed as a useful commodity in our theorizing if the theory is to hold water. The extended model responds, to some degree, to an interpretation of Peirce that attempts to make sense of a wholesale metaphysical system starting from the point of semiotics (as an institutional and research practice), with examples such as the idea of a semiotic fundamental law (Hulswit, Romanini 2014) and the thorough commitment that comes with such an approach. Peirce, however, is not the only reason for us to have such explanatory power. ${ }^{5}$ There is an argument to be made regarding the idea that semiotics can make discoveries, scientific or otherwise. If semiotics is capable of positioning itself as a fundamental kind of research, its area of research becomes far more open by default, its claims offering not only a novel insight about meaning in general, but about what other sciences can claim about the world.

Can semiotic theories actually have that level of explanatory power? Is the extended model the correct way forward for semiotic research? My contention is that it can't, and it isn't. While there is no harm done in exploring the possibility that it may, there are issues in how to make it work. For starters, having claims about meaning at a fundamental level causing other forms of meaning implies a non-trivial connection from bottom to top, ${ }^{6}$ meaning hinging upon meaning upon meaning. But the way to have physical constraints, following Salthe's terminology, as actual meaningful entities lies in the assumption that these must be meaningful on their own. The argument for vis a prospecto becomes substantially more tempting as a way to connect bottom to top without having to deliver strong explanations as for why physical constraints would entail meaning by themselves, but this requires the a priori assumption that future general forms of meaning can indeed interact with previous forms in a direct fashion. As far as evidence goes, this seems to be the case of a commitment, not an explanation.

Such a problem takes us to another point: Can we make semiotic discoveries and give semiotic explanations based on the premises of the extended model? The main issue to be found with the extended model (if we accept my description along general lines) lies in the foundational approach, the lawful recognition of semiosis as an elementary force. But where does that take us? If meaning is universally lawful, does that change our description of physical systems? The answer is probably 'no,' though the challenge would be to give a positive account of it. Now, in his critique to Peircean biosemiotics, Barbieri points to the explanatory inefficacy of (Peircean) semiotic concepts when applied to biological interactions, considering them simply a renaming of elements that brings forth no new knowledge about them (Barbieri 2015, 167).

Now, I will, for the time being, put explanations and discoveries in the same bag as a way to briefly treat the problem described by Barbieri. There may be differences to be accounted for in a more substantial analysis, but we can assume both of these are related insofar as the expectation for consequential semiotic statements is high. What would count as a semiotic explanation would then be a consequential semiotic statement about some phenomenon, and what would count as a semiotic discovery would come about as a consequential finding related to semiotic explanations opening a heretofore unknown space about some phenomenon.

Would universal statements about the evolution of semiosis from nothing to culture give us non-trivial explanations or discoveries? If we state that there may be sign relations entailed purely by physical systems, we would have either a case of a fundamental discovery, or of a trivial explanation. If we think it is the case that sign relations in physical systems are a semiotic discovery (as opposed to a terminological description that adds nothing to the mix), then the consequences may indeed come to impact how we talk about semiosis in humans. However, the contention lies in that there does not seem to be an actual change in things. If we frame physical interactions as relations, the only gain in terms of discoveries is allowing ourselves to state that, based on this premise, other possibilities may arise. In other words, as far as discoveries go, this would only remain as an a priori statement, allowing new potential discoveries.

Can semiotic theories be universalistic in their scope? If we think of them as capable of explaining such a wide range of phenomena including what lies below the standard threshold, then the answer would be positive. And yet, the option remains descriptive in that the use of semiotic phenomena at that point remains useful only for allowing other semiotic theories to stand as long as we accept the previous ones. The criticism Barbieri has raised is not circumvented.

If semiotics, particularly in its biosemiotics strand, can stand as a philosophical system, then there is the very real possibility that there is no need for semiotic discoveries, but the philosophical branch of biosemiotics seems to be at odds with the more practical areas of its research. The posited universalism of the extended model doesn't carry to the application of semiotic methods, and one could argue that the most prominent points where biosemiotics has been an effective

\footnotetext{
5 There are even some grounds for coining a "post-Peircean" biosemiotics (Rodríguez Higuera 2020).

6 I understand "trivial" here as the same idea that we can have a sign entailed with an abiotic object without making the sign fully abiotic. In other words, a trivial connection in this sense would simply be the fact that when something has some physical form, this physical form does not entail the totality of facts about the meaningful interaction in question.
} 
force of change in how we think about biology rests in the more constrained views on semiosis (that is, over the standard threshold). Again, there is nothing wrong with attempting to expand towards further areas and examine the possibility of semiosis, but its possibility does not entail necessity or actuality, and the extended model of semiosis seems to fail at giving a coherent account of semiosis in favor of one that would net the most discoveries, however egregious those could be.

\section{ACKNOWLEDGEMENTS}

This research was supported by the Support of Academic Mobility project at Palacký University Olomouc, project number CZ.02.2.69/0.0/0.0/16_027/0008482.

\section{REFERENCES}

Barbieri, M., 2007. Is The Cell A Semiotic System? In Barbieri, M. (Ed.), Introduction to Biosemiotics. Dordrecht: Springer Netherlands, pp. 179-208.

Barbieri, M., 2015. Code Biology: A New Science of Life. Cham: Springer.

Deely, J., 2001. Physiosemiosis in the Semiotic Spiral: A Play of Musement. Sign Systems Studies, 29(1), 27-47.

Deely, J. N., 2012. Vis a Prospecto. In Favareau, D. F., Cobley, P., Kull, K. (Eds.), A More Developed Sign: Interpreting the Work of Jesper Hoffmeyer. Tartu Semiotics Library, 10, Tartu: University of Tartu Press, pp. 315-318.
Hulswit, M., Romanini, V., 2014. Peircean habits, broken symmetries, and biosemiotics. In Romanini, V., Fernández, E. (Eds.), Peirce and Biosemiotics: A Guess at the Riddle of Life, Biosemiotics, 10, Dordrecht: Springer, pp. 95-126.

Koch, W., 1986. Evolutionary Cultural Semiotics. Bochum Publications in Evolutionary Cultural Semiotics, 6, Bochum: Brockmeyer.

Kull, K., Emmeche, C., Hoffmeyer, J., 2011. Why Biosemiotics? An Introduction to Our View on the Biology of Life Itself. In Emmeche, C., Kull, K. (Eds.) Towards a Semiotic Biology. London: Imperial College Press, pp. 1-21.

Rodríguez Higuera, C. J., Kull, K., 2017. The Biosemiotic Glossary Project: The Semiotic Threshold. Biosemiotics, 10(1), 109-126.

Rodríguez Higuera, C. J., 2020. Everything seems so settled here: The conceivability of post-Peircean biosemiotics. Sign Systems Studies 47(3/4), 420-435.

Salthe, S., 2007. Meaning in Nature: Placing Biosemiotics within Pansemiotics. In Barbieri, M. (Ed.), Biosemiotics: Information, Codes and Signs in Living Systems. New York: Nova Science, pp. 207-217.

Salthe, S., 2008. What is the scope of biosemiotics? Information in living systems. In Barbieri, M. (Ed.), Introduction to Biosemiotics: The New Biological Synthesis. Dordrecht: Springer, pp. 133-148.

Salthe, S., 2012. On the Origin of Semiosis. Cybernetics and Human Knowing. 19(3), 53-66.

Sharov, A. A., Vehkavaara, T., 2015. Protosemiosis: agency with reduced representation capacity. Biosemiotics, 8(1), 103-123. 